\title{
Review
}

\section{An overview of SARS-CoV-2 and animal infection}

\section{Mohamed A. A. MAHDY}

${ }^{a}$ Department of Anatomy and Embryology, Faculty of Veterinary Medicine, South Valley University, Qena 83523, Egypt

Correspondence: Mohamed A. A. Mahdy,

Department of Anatomy and Embryology,

Faculty of Veterinary Medicine,

South Valley University,

Qena 83523, Egypt

Tel: +2010716280 Fax: +20963211281

E-Mail: m_mahdi@vet.svu.edu.eg

https://orcid.org/0000-0001-6402-2944 


\begin{abstract}
A novel coronavirus has been reported as the causative pathogen of the Coronavirus disease 2019 (COVID-19) outbreak Wuhan city, China in December 2019. Due to the rapid spreading of COVID-19 worldwide, it has been announced as a pandemic by the World Health Organization (WHO). Hospitalized patients in Wuhan are associated with the Huanan seafood wholesale market where live animals, such as poultry, bats, snakes, frogs, rabbits, marmots, and hedgehogs are sold in that market which suggests a possible zoonotic infection. Therefore, it is essential to identify the potential animal reservoir, and the possibility of infection for other animal species. This short review aims to provide an overview on the relation between severe acute respiratory syndrome coronavirus-2 (SARS-CoV-2) infection and animals.
\end{abstract}

\title{
Keywords: Animal, COVID-19, intermediate host, SARS-COV-2
}




\section{Introduction}

A novel coronavirus, severe acute respiratory syndrome coronavirus-2 (SARS-CoV-2), has been reported as the causative pathogen of the Coronavirus disease 2019 (COVID-19) outbreak, which later has been announced as COVID-19 pandemic by the World Health Organization (WHO). COVID-19 pandemic is still spreading rapidly worldwide in more than 213 countries with 1,918,138 confirmed cases and 123,126 mortalities, as of April 10, 2020 [1].

Coronaviruses (CoVs) has four genera that infect a variety of domestic and wild animals, as well as humans. [2]. Mammals, specifically bats, are the natural hosts of Alpha ( $\alpha)$ - and $\beta$-CoVs, while pigs and birds are the natural hosts of gamma $(\gamma)$ - and delta $(\delta)-\mathrm{CoVs}[3]$. CoVs have great ability to mutate which facilitate their transmission from animals to humans [4].

SARS-CoV-2 is a novel betacoronavirus ( $\beta$-coronavirus) belongs to the subgenus sarbecovirus of the Coronaviridae family. It is firstly identified in hospitalized patients in Wuhan city, Hubei Province, China in December 2019 [5]. Those patients are associated with the Huanan seafood wholesale market where live animals, such as poultry, bats, snakes, frogs, rabbits, marmots, and hedgehogs are sold in that market suggesting a possible zoonotic infection [6]. Therefore, it is essential to identify the potential virus reservoir, and the possibility of infection for other animal species. This short review aims to provide an overview on the relation between SARS-CoV-2 and animals.

\section{Possible animal reservoir:}

Bats:

SARS-CoV-2 is about $96.2 \%$ identical to the genetic information of the bat SARS-like CoV (RaTG13) based on genome sequencing and evolutionary analysis [5, 7-9], which suggests the 
possibility that SARS-CoV-2 originates from bats [8-10]. Due to the lack of direct contact between bats and humans, direct human infection with bat CoVs is rare. It is assumed that transmission of SARS-CoV-2 from bats to human passes through an unknown intermediate host that might facilitates its transfer to human [7-10]. The analysis of genomic sequences of CoVs suggests that SARS-CoV-2 is a recombinant virus that arise between the bat $\mathrm{CoV}$ and another coronavirus of unknown origin [7]. Meanwhile, mutation in Spike Glycoprotein (S) and nucleocapsid protein $(\mathrm{N})$ of SARS-CoV-2 makes it distinct from bat SARS-like CoV supporting the hypothesis that SARS-CoV-2 mutates before its transmission from bats and acquire its ability to infect human [9]. SARS-CoV-2 invades cells mainly through binding of the virus $\mathrm{S}$ protein and host cell receptor, angiotensin-converting enzyme 2, (ACE2) [11, 12]. Although, SARSCoV-2 recognizes ACE2 from a variety of animal species, including palm civet, SARS-CoV-2 acquires the capability to infect humans, as well as, to transmit among humans [12]. The binding affinity of SARS-CoV-2 to human ACE2 is higher than that of SARS-CoV [11, 12] which suggests a possibility of cross-species transmission [13] and the rapid spread of this virus [11, 12]. On the other hand, Friedrich-Loeffler-Institut (FLI) in Germany reported that experimentally infected Egyptian fruit bats show no symptoms of disease, moreover, they do not infect other bats [14].

\section{Pangolin:}

The whole genome of pangolin-CoV is highly similar to that of the SARS-CoV-2 and bat CoV [15]. However, the similarity between pangolin-CoV and SARS-CoV-2 is higher than that with the bat $\mathrm{CoV}[16,17]$. Moreover, pangolin CoVs share 5 identical amino acids with SARS-CoV-2 whereas bat CoV RaTG13 only shares one amino acid with SARS-CoV-2 [18]. Furthermore, the 
receptor-binding domain (RBD) of the pangolin-CoV is nearly identical to that of SARS-CoV-2 with strong binding ability to human ACE2 indicating that pangolin is involved in the recombination of SARS-CoV-2 [15]. However, the evidence of phylogenetic analyses support that the 2019-nCoV did not arise directly from the pangolin-CoV [17]. These results suggest that pangolin could be a potential intermediate host for SARS-CoV-2 [18].

\section{Reptiles:}

Liu et al. [19] reported that turtles (Chrysemys picta bellii, Pelodiscus sinensis, and Chelonia mydas) might act as one of the potential intermediate hosts that transmit SARS-CoV-2 to humans. Moreover, evolutionary analysis, as well as analysis of the codon usage of SARS-CoV2 suggest that snake might be potential wildlife animal reservoir for SARS-CoV-2 [7, 19]. However, ACE2 of turtle and snake loses its capability to bind to S protein of SARS-CoV-2, therefore these reptiles should not be considered as potential hosts for SARS-CoV-2 [20].

\section{SARS-CoV-2 and animal infection:}

The hospitalized patients in Wuhan are associated with the Huanan seafood wholesale market. In this market, live animals, such as poultry, bats, snakes, frogs, rabbits, marmots, and hedgehogs are sold which suggests a possible zoonotic infection [6]. It is critical to understand the susceptibility of animals to SARS-CoV-2 in order to control COVID-19. Until now, there is no evidence that animals have a role in the spread of the SARS-CoV-2 pandemic [21].

\section{Cats:}

SARS-CoV-2 infects cat populations in Wuhan during the COVID-19 outbreak based on detection of SARS-CoV-2 antibodies in those cats. Infection to those cats arise upon contact with COVID-19 patients or SARS-CoV-2 polluted environment [22]. In addition, SARS-CoV-2 has been detected in the feces and vomit of an infected pet cat with COVID-19 in Belgium indicating 
active replication of the virus [23]. In this respect, SARS-CoV-2 has been reported to replicate only in the upper respiratory tract of cats and this replication is not associated with severe disease or death [24]. It is worth to mention that younger cats are more tolerant to SARS-CoV-2 infection [24].

\section{Dogs:}

Although dogs have low susceptibility to SARSCoV-2 infection [24], pet dogs in Hong Kong and North Italy have been infected with SARSCoV-2 infection, without symptoms, due to contact with COVID-19 infected persons [13, 25]. Dogs have an ACE2 receptors, similar to human ACE2, that function as SARS-CoV receptors which raises the possibility that dogs might be a potential intermediate host [13].

\section{Other animals:}

A Tiger at Bronx Zoo, New York, USA has been tested positive for COVID-19 as the first case of animal infection in USA. The tiger receives infection from an infected employee [26]. On the contrary, chickens, ducks, and pigs are not susceptible to SARS-CoV-2 infection [14, 24].

\section{Animal models for studying SARS-CoV-2:}

There is an urgent need for animal models that mimic the clinical and pathological characteristics of human SARS-CoV-2 infection to help studying its pathogenesis. These models contribute to assess the efficacy and safety of the novel antiviral treatment and vaccine trials [27]. The candidate animal model should presents the active infection, virus shedding, and transmission to naïve animals [28].

\section{Rodents:}


SARS-CoV-2 has very low binding affinity to ACE2 of mice compared with that in human. Due to the inefficient replication in mice, they cannot be used as animal models to test vaccine or antiviral drugs [29]. However, virus replication has been reported in lungs of hACE2 transgenic mice without clear symptoms after SARS-CoV-2 infection, therefore, this model can be used in therapeutic and vaccine studies of SARS-CoV-2 [30].

Both the clinical and histopathological findings of the SARS-CoV-2-infeted hamster closely resemble the manifestations of human upper and lower respiratory tract infection with virus shedding in respiratory droplets and faeces. Additionally, the virus transmits to naïve contact hamsters in close contact with infected ones. Furthermore, Syrian hamster ACE2 protein shows a high binding affinity to the Spike of SARS-CoV-2. Therefore, Syrian hamster model could be a suitable model simulating the clinical, pathological, virological, and immunological features of SARS-CoV-2-induced COVID-19 [27].

\section{Cats:}

Since SARS-CoV-2 replicates well in the upper respiratory tract of cats. In addition, SARSCoV-2 transmits from an infected cat to non-infected one through direct contact with respiratory droplets [24]. Virus shedding has been reported in the feces of an infected pet cat with COVID19 in Belgium [23]. Therefore, cats also could be used as an optimal animal model for screening antiviral drugs or vaccines against SARS-CoV-2 [29].

\section{Ferrets:}

Ferrets are frequently used as an animal model for studying human respiratory viral infection [31]. Ferrets are highly susceptible to infection with SARS-CoV-2 [14, 28]. The virus replicates in the upper respiratory tract with high virus titer in the upper respiratory tract associated with virus shedding in urine and fecal samples. Infected ferrets show acute bronchiolitis without 
causing severe disease or death [24, 28]. Additionally, SARS-CoV-2 actively transmits from infected ferrets to naïve ones through direct contact and occasionally airborne transmission. Infected ferrets are capable to transmit the virus at 2 days post infection before reaching the peak of viral RNA copy number and the peak of body temperature as well [28]. Therefore, ferrets can be used as animal models for assessing antiviral drugs or vaccine against SARS-CoV-2 [14, 28]. However, infected ferrets show some limitations, such as the mild symptoms and low virus titer in lungs [28].

\section{Primates:}

Young, adult, and aged infected cynomolgus macaques express viral RNA in nasal swabs without developing clinical symptoms. However, aged animals express higher level of viral RNA and prolonged virus shedding compared to young and adult animals [32]. On the other hand, infected rhesus macaques show weight loss with rapid respiration associated with moderate interstitial pneumonia and virus replication both in the upper and lower respiratory tract. Neither clinical symptoms nor virus replication is detected in recovered monkeys exposed to a second infection with SARS-CoV-2. These results indicate that primary infected monkeys develop immunity against SARS-CoV-2 that protects those monkeys from the second infection [33]. Therefore, rhesus macaque is a suitable animal model to evaluate vaccine safety.

\section{Precautions on pet animals}

Although there is no confirmation that pet animals have a role in spreading of the SARS-CoV-2 pandemic, researchers suggest that pet animals may be susceptible to SARS-CoV-2 infection. Therefore, it is recommended to limit the contact between the infected owner and pets, avoid 
kissing animals, and applying basic hygiene measures [21]. Additionally, pet animals exposed to COVID-19 patients should be screened for SARS-CoV-2 [29].

\section{Concluding remarks:}

This review provides an overview of the current knowledge of the relation between SARS-CoV-2 infection and the role played by animals. SARS-CoV-2 originates from bats and passes through an unknown intermediate host, which facilitates its transfer to human. Although it is not confirmed that pet animals play a role in spreading of the SARS-CoV-2 pandemic, some animals receive SARS-CoV-2 infection upon contact with infected individual. Therefore, it is recommended to take apply basic hygiene measures to limit the contact between the infected patients and pets. Several animal models have been reported as candidates for assessing the efficacy and safety of antiviral drugs or testing experimental vaccine against SARS-CoV-2 including hACE2 transgenic mice, cats, and ferrets.

\section{REFERENCES:}

1. WHO. 2019. Coronavirus disease (COVID-19) Pandemic [Online]. World Health Organization. Available: https://www.who.int/emergencies/diseases/novel-coronavirus$\underline{2019}$ [Accessed 15/04 2020].

2. Fan, Y., Zhao, K., Shi, Z.-L. and Zhou, P. 2019. Bat Coronaviruses in China. Viruses 11: 210; Seah, I. and Agrawal, R. 2020. Can the coronavirus disease 2019 (COVID-19) affect the eyes? A review of coronaviruses and ocular implications in humans and animals. Ocul Immunol Inflamm: 1-5.

3. Velavan, T. P. and Meyer, C. G. 2020. The COVID-19 epidemic. Tropical Medicine \& International Health 25: 278-280. 
4. Woo, P. C., Lau, S. K., Huang, Y. and Yuen, K. Y. 2009. Coronavirus diversity, phylogeny and interspecies jumping. Exp Biol Med (Maywood) 234: 1117-1127.

5. Zhu, N., Zhang, D., Wang, W., Li, X., Yang, B., Song, J., Zhao, X., Huang, B., Shi, W., Lu, R., Niu, P., Zhan, F., Ma, X., Wang, D., Xu, W., Wu, G., Gao, G. F. and Tan, W. 2020. A novel coronavirus from patients with pneumonia in China, 2019. N Engl J Med 382: 727733.

6. Malik, Y. S., Sircar, S., Bhat, S., Vinodhkumar, O. R., Tiwari, R., Sah, R., Rabaan, A. A., Rodriguez-Morales, A. J. and Dhama, K. 2020. Emerging Coronavirus Disease (COVID19), a pandemic public health emergency with animal linkages: Current status update.

7. Ji, W., Wang, W., Zhao, X., Zai, J. and Li, X. 2020. Cross-species transmission of the newly identified coronavirus 2019-nCoV. J Med Virol 92: 433-440.

8. Zhou, P., Yang, X.-L., Wang, X.-G., Hu, B., Zhang, L., Zhang, W., Si, H.-R., Zhu, Y., Li, B., Huang, C.-L., Chen, H.-D., Chen, J., Luo, Y., Guo, H., Jiang, R.-D., Liu, M.-Q., Chen, Y., Shen, X.-R., Wang, X., Zheng, X.-S., Zhao, K., Chen, Q.-J., Deng, F., Liu, L.-L., Yan, B., Zhan, F.-X., Wang, Y.-Y., Xiao, G.-F. and Shi, Z.-L. 2020. A pneumonia outbreak associated with a new coronavirus of probable bat origin. Nature 579: 270-273.

9. Benvenuto, D., Giovanetti, M., Ciccozzi, A., Spoto, S., Angeletti, S. and Ciccozzi, M. 2020. The 2019-new coronavirus epidemic: Evidence for virus evolution. J Med Virol 92: 455459.

10. Guo, Y.-R., Cao, Q.-D., Hong, Z.-S., Tan, Y.-Y., Chen, S.-D., Jin, H.-J., Tan, K.-S., Wang, D.-Y. and Yan, Y. 2020. The origin, transmission and clinical therapies on coronavirus disease 2019 (COVID-19) outbreak - an update on the status. Military Medical Research 7: 11-11. 
11. Ortega, J. T., Serrano, M. L., Pujol, F. H. and Rangel, H. R. 2020. Role of changes in SARS-CoV-2 spike protein in the interaction with the human ACE2 receptor: An in silico analysis. EXCLI journal 19: 410-417.

12. Wan, Y., Shang, J., Graham, R., Baric, R. S. and Li, F. 2020. Receptor recognition by the novel coronavirus from Wuhan: an analysis based on decade-long structural studies of SARS coronavirus. J Virol 94: e00127-00120.

13. Goumenou, M., Spandidos, D. A. and Tsatsakis, A. 2020. Possibility of transmission through dogs being a contributing factor to the extreme Covid-19 outbreak in North Italy. Mol Med Rep.

14. FLI 2020. Press Release: Novel Coronavirus SARS-CoV-2: Fruit bats and ferrets are susceptible, pigs and chickens are not. Greifswald - Insel Riems: Friedrich-LoefflerInstitut.

15. Xiao, K., Zhai, J., Feng, Y., Zhou, N., Zhang, X., Zou, J.-J., Li, N., Guo, Y., Li, X., Shen, X., Zhang, Z., Shu, F., Huang, W., Li, Y., Zhang, Z., Chen, R.-A., Wu, Y.-J., Peng, S.M., Huang, M., Xie, W.-J., Cai, Q.-H., Hou, F.-H., Liu, Y., Chen, W., Xiao, L. and Shen, Y. 2020. Isolation and characterization of 2019-nCoV-like coronavirus from Malayan Pangolins. bioRxiv: 2020.2002.2017.951335.

16. Zhang, T., Wu, Q. and Zhang, Z. 2020. Pangolin homology associated with 2019-nCoV. bioRxiv: 2020.2002.2019.950253.

17. Liu, P., Jiang, J.-Z., Wan, X.-F., Hua, Y., Wang, X., Hou, F., Chen, J., Zou, J. and Chen, J. 2020. Are pangolins the intermediate host of the 2019 novel coronavirus (2019-nCoV) ? bioRxiv: 2020.2002.2018.954628. 
18. Lam, T. T., Shum, M. H., Zhu, H. C., Tong, Y. G., Ni, X. B., Liao, Y. S., Wei, W., Cheung, W. Y., Li, W. J., Li, L. F., Leung, G. M., Holmes, E. C., Hu, Y. L. and Guan, Y. 2020. Identifying SARS-CoV-2 related coronaviruses in Malayan pangolins. Nature.

19. Liu, Z., Xiao, X., Wei, X., Li, J., Yang, J., Tan, H., Zhu, J., Zhang, Q., Wu, J. and Liu, L. 2020. Composition and divergence of coronavirus spike proteins and host ACE2 receptors predict potential intermediate hosts of SARS-CoV-2. J Med Virol n/a: 1-7.

20. Luan, J., Jin, X., Lu, Y. and Zhang, L. 2020. SARS-CoV-2 spike protein favors ACE2 from Bovidae and Cricetidae. J Med Virol n/a.

21. Agrimi, U. 2020. SARS-CoV-2 infection among domestic animals [Online]. Epidemiology for public health, Istituto Superiore di Sanità. Available: https://www.epicentro.iss.it/en/coronavirus/sars-cov-2-domestic-animals [Accessed 08/04 2020].

22. Zhang, Q., Zhang, H., Huang, K., Yang, Y., Hui, X., Gao, J., He, X., Li, C., Gong, W., Zhang, Y., Peng, C., Gao, X., Chen, H., Zou, Z., Shi, Z. and Jin, M. 2020. SARS-CoV-2 neutralizing serum antibodies in cats: a serological investigation. bioRxiv: 2020.2004.2001.021196.

23. Thompson, H. 2020. A cat appears to have caught the coronavirus, but it's complicated. Science News, 31/03/2020, p.08/04.

24. Shi, J., Wen, Z., Zhong, G., Yang, H., Wang, C., Huang, B., Liu, R., He, X., Shuai, L., Sun, Z., Zhao, Y., Liu, P., Liang, L., Cui, P., Wang, J., Zhang, X., Guan, Y., Tan, W., Wu, G., Chen, H. and Bu, Z. 2020. Susceptibility of ferrets, cats, dogs, and other domesticated animals to SARS-coronavirus 2. Science: eabb7015.

25. Loeb, J. 2020. Pet dog confirmed to have coronavirus. Vet Rec 186: 265. 
26. WCS 2020. A Tiger at Bronx Zoo tests positive for COVID-19; The tiger and the zoo's other cats are doing well at this time. Wildlife Conservation Society.

27. Chan, J. F.-W., Zhang, A. J., Yuan, S., Poon, V. K.-M., Chan, C. C.-S., Lee, A. C.-Y., Chan, W.-M., Fan, Z., Tsoi, H.-W., Wen, L., Liang, R., Cao, J., Chen, Y., Tang, K., Luo, C., Cai, J.-P., Kok, K.-H., Chu, H., Chan, K.-H., Sridhar, S., Chen, Z., Chen, H., To, K. K.-W. and Yuen, K.-Y. 2020. Simulation of the clinical and pathological manifestations of Coronavirus Disease 2019 (COVID-19) in golden Syrian hamster model: implications for disease pathogenesis and transmissibility. Clinical Infectious Diseases.

28. Kim, Y.-I., Kim, S.-G., Kim, S.-M., Kim, E.-H., Park, S.-J., Yu, K.-M., Chang, J.-H., Kim, E. J., Lee, S., Casel, M. A. B., Um, J., Song, M.-S., Jeong, H. W., Lai, V. D., Kim, Y., Chin, B. S., Park, J.-S., Chung, K.-H., Foo, S.-S., Poo, H., Mo, I.-P., Lee, O.-J., Webby, R. J., Jung, J. U. and Choi, Y. K. 2020. Infection and rapid transmission of SARS-CoV-2 in ferrets. Cell Host \& Microbe.

29. Shen, M., Liu, C., Xu, R., Ruan, Z., Zhao, S., Zhang, H., Wang, W., Huang, X., Yang, L., Tang, Y., Yang, T. and Jia, X. 2020. SARS-CoV-2 Infection of Cats and Dogs? Preprints, 2020040116.

30. Bao, L., Deng, W., Huang, B., Gao, H., Ren, L., Wei, Q., Yu, P., Xu, Y., Liu, J., Qi, F., Qu, Y., Wang, W., Li, F., Lv, Q., Xue, J., Gong, S., Liu, M., Wang, G., Wang, S., Zhao, L., Liu, P., Zhao, L., Ye, F., Wang, H., Zhou, W., Zhu, N., Zhen, W., Zhang, X., Song, Z., Guo, L., Chen, L., Wang, C., Wang, Y., Wang, X., Xiao, Y., Sun, Q., Liu, H., Zhu, F., Ma, C., Yan, L., Yang, M., Han, J., Xu, W., Tan, W., Peng, X., Jin, Q., Wu, G. and Qin, C. 2020. The pathogenicity of 2019 novel coronavirus in hACE2 transgenic mice. bioRxiv: 2020.2002.2007.939389. 
31. Stittelaar, K. J., de Waal, L., van Amerongen, G., Veldhuis Kroeze, E. J., Fraaij, P. L., van Baalen, C. A., van Kampen, J. J., van der Vries, E., Osterhaus, A. D. and de Swart, R. L. 2016. Ferrets as a novel animal model for studying human respiratory syncytial virus infections in immunocompetent and immunocompromised hosts. Viruses 8; Zhang, Q., Shi, J., Deng, G., Guo, J., Zeng, X., He, X., Kong, H., Gu, C., Li, X., Liu, J., Wang, G., Chen, Y., Liu, L., Liang, L., Li, Y., Fan, J., Wang, J., Li, W., Guan, L., Li, Q., Yang, H., Chen, P., Jiang, L., Guan, Y., Xin, X., Jiang, Y., Tian, G., Wang, X., Qiao, C., Li, C., Bu, Z. and Chen, H. 2013. H7N9 influenza viruses are transmissible in ferrets by respiratory droplet. Science 341: 410-414.

32. Rockx, B., Kuiken, T., Herfst, S., Bestebroer, T., Lamers, M. M., de Meulder, D., van Amerongen, G., van den Brand, J., Okba, N. M. A., Schipper, D., van Run, P., Leijten, L., Verschoor, E., Verstrepen, B., Langermans, J., Drosten, C., van Vlissingen, M. F., Fouchier, R., de Swart, R., Koopmans, M. and Haagmans, B. L. 2020. Comparative pathogenesis of COVID-19, MERS And SARS in a non-human primate model. bioRxiv: 2020.2003.2017.995639.

33. Bao, L., Deng, W., Gao, H., Xiao, C., Liu, J., Xue, J., Lv, Q., Liu, J., Yu, P., Xu, Y., Qi, F., Qu, Y., Li, F., Xiang, Z., Yu, H., Gong, S., Liu, M., Wang, G., Wang, S., Song, Z., Zhao, W., Han, Y., Zhao, L., Liu, X., Wei, Q. and Qin, C. 2020. Reinfection could not occur in SARS-CoV-2 infected rhesus macaques. bioRxiv: 2020.2003.2013.990226. 